\title{
Analytical and Experimental Studies of Leak Location and Environment Characterization for the International Space Station
}

Michael Woronowicz ${ }^{\mathrm{a}}$, Joshua Abel ${ }^{\mathrm{b}}$, David Autrey ${ }^{\mathrm{c}}$, Rebecca Blackmon ${ }^{\mathrm{a}}$, Tim Bond ${ }^{\mathrm{d}}$, Martin Brown ${ }^{\mathrm{a}}$, Jesse Buffington ${ }^{\mathrm{d}}$, Edward Cheng ${ }^{\mathrm{e}}$, Danielle DeLatte ${ }^{\mathrm{f}}$, Kelvin Garcia $^{\mathrm{g}}$, Jodie Glenn ${ }^{\mathrm{c}}$, Doug Hawk ${ }^{\mathrm{b}}$, Jonathan Ma e, Jelila Mohammed ${ }^{\mathrm{g}}$, Kristina Montt de Garcia ${ }^{\mathrm{g}}$, Radford Perry ${ }^{\mathrm{g}}$, Dino Rossetti ${ }^{\mathrm{e}}$, Kimathi Tull ${ }^{\mathrm{h}}$, Eric Warren ${ }^{\mathrm{c}}$

${ }^{a}$ Stinger Ghaffarian Technologies, Inc

${ }^{b} A T K$

c Wyle STE Group

${ }^{d}$ NASA Johnson Space Flight Center

e Conceptual Analytics

${ }^{f}$ ASRC Federal Space \& Defense

g NASA Goddard Space Flight Center

h Jackson and Tull 


\section{presented by \\ Prof. Chunpei Cai \\ New Mexico State University}




\section{Outline}

- Introduction

- Analytical Approach

- Instrument Features

- Discussion of Similar Spaceflight Applications

- Test Setup

- Configuration

- Types of leak sources

- Results

- Selected case measurements

- Model comparison 


\section{Introduction}

- International Space Station (ISS) program officials have long been considering ways to locate external gaseous leaks from various sources

- $\mathrm{NH}_{3}$ from radiator assemblies

- Air from crew compartments

- Crew members have performed two unplanned spacewalks since October 2012 to isolate $\mathrm{NH}_{3}$ leaks from a Thermal Control System on the P6 Truss Assembly 


\section{(2) 사요}

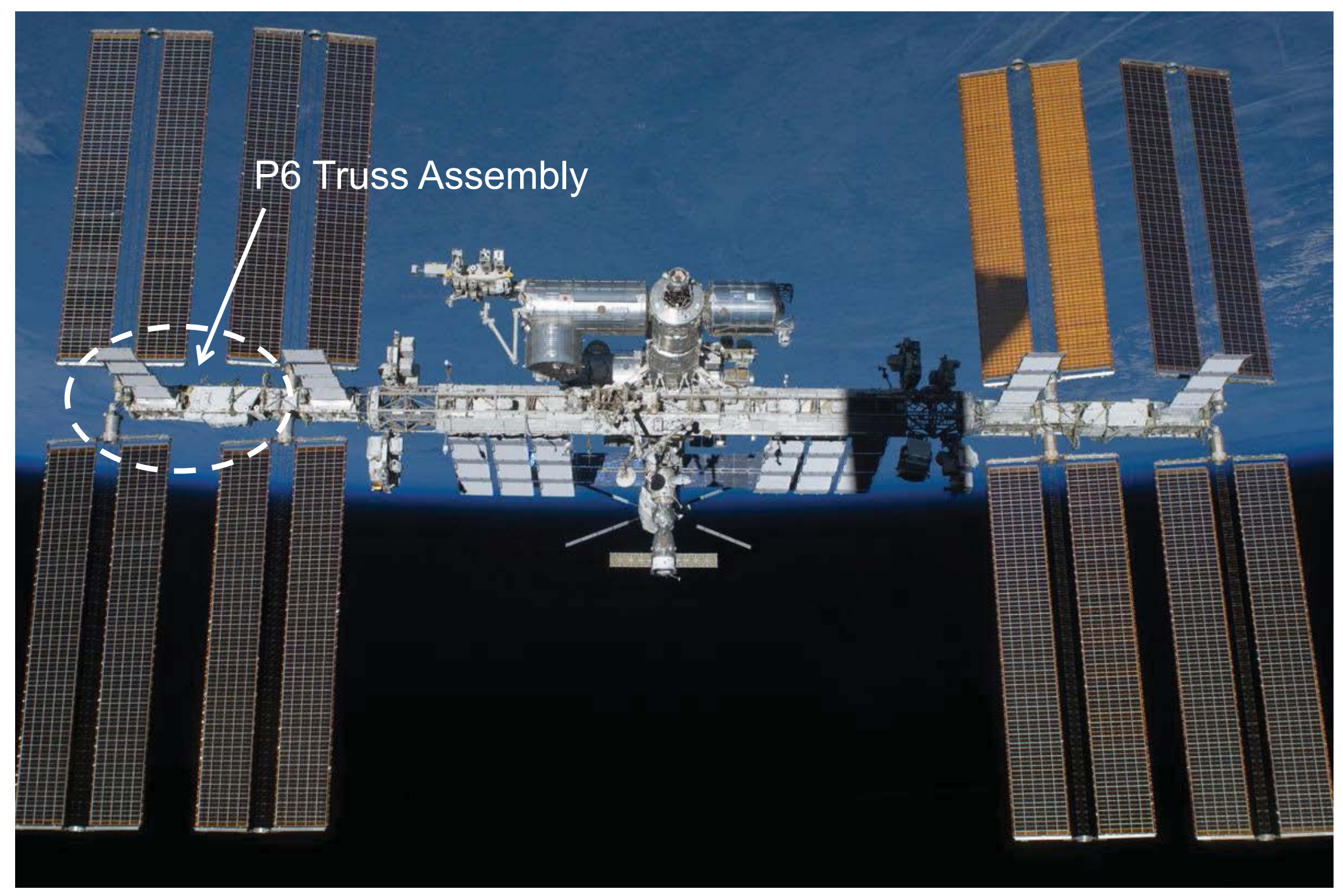




\section{Introduction (3 of 3)}

- ISS program is developing a robotically-operated leak locator tool featuring commercial off-the-shelf (COTS) units

- Residual gas analyzer (RGA) for partial pressure measurements

- Faint, distant, below ambient environment $\left(10-10^{3} \mathrm{lb}_{\mathrm{m}} / \mathrm{yr}\right.$.)

- Full range gauge (FRG) for total pressure measurements

- Robust, proximal, above ambient environment (10-50 $\mathrm{lb}_{\mathrm{m}} /$ day)

- Called the Ammonia Leak Locator-ALL

- ALL would be positioned, oriented in various ways near suspect surfaces, use data to determine leak locations

- Test activity focus

- Sensitivity (minimum detection level, directional influence) 


\section{Objective}

- Compare RGA performance during ground testing to results from analytical plume model

- Assess suitability for providing model predictions during on-orbit operations to assist with leak location 


\section{Source Behavior}

- External neutral gas phase sources on ISS result from a number of different physical mechanisms

- Supersonic expansion through thruster nozzles

- Pressure-driven acceleration to sonic conditions across an orifice

- Surface evaporation (may or may not have bulk velocity)

- Effusion $(M=0)$

- Effusion also often describes equilibrium gas conditions at rest

- e.g. flux to a surface within a vacuum chamber

- Mass flow rate $\dot{m}$ and pressure related by Hertz-Knudsen equation

$$
\dot{m}=\rho A \sqrt{\frac{R T}{2 \pi}}=\frac{p A}{\sqrt{2 \pi R T}}
$$




\section{Plume Model Description}

- Can consider modeling this regime using free molecule flow

- Collision rates fall rapidly with distance from source

- Majority of self-scattering collisions occur when faster molecules overtake slower ones on similar trajectories from source

- Center-of-mass motion remains unchanged during collision

- Existence of collisions may not substantially alter plume distribution from free-molecule description

- Have demonstrated considerable levels of success with such an approach over a wide range of applications 


\section{Plume Model Formulation-Source}

- Find particular solution to collisionless Boltzmann equation for source $Q_{1}$ :

$$
\frac{\partial f}{\partial t}+\boldsymbol{v} \cdot \frac{\partial f}{\partial \boldsymbol{x}}+\boldsymbol{g} \cdot \frac{\partial f}{\partial \boldsymbol{v}}=Q_{1}
$$

where $Q_{1}$ represents a Lambertian source superimposed on a bulk velocity

$$
Q_{1} \equiv \frac{2 \beta^{4}}{A_{1} \pi} \delta(\boldsymbol{x}) \dot{m}(t)|\boldsymbol{v} \cdot \hat{\boldsymbol{n}}| \exp \left(-\beta^{2}\left(\boldsymbol{v}-\boldsymbol{u}_{\mathrm{e}}\right)^{2}\right)
$$

and the normalization factor is given by

$$
A_{1} \equiv e^{-s^{2} \cos ^{2} \phi_{\mathrm{e}}}+\sqrt{\pi} s \cos \phi_{\mathrm{e}}\left(1+\operatorname{erf}\left(s \cos \phi_{\mathrm{e}}\right)\right)
$$




\section{Plume Model Formulation-Definitions}
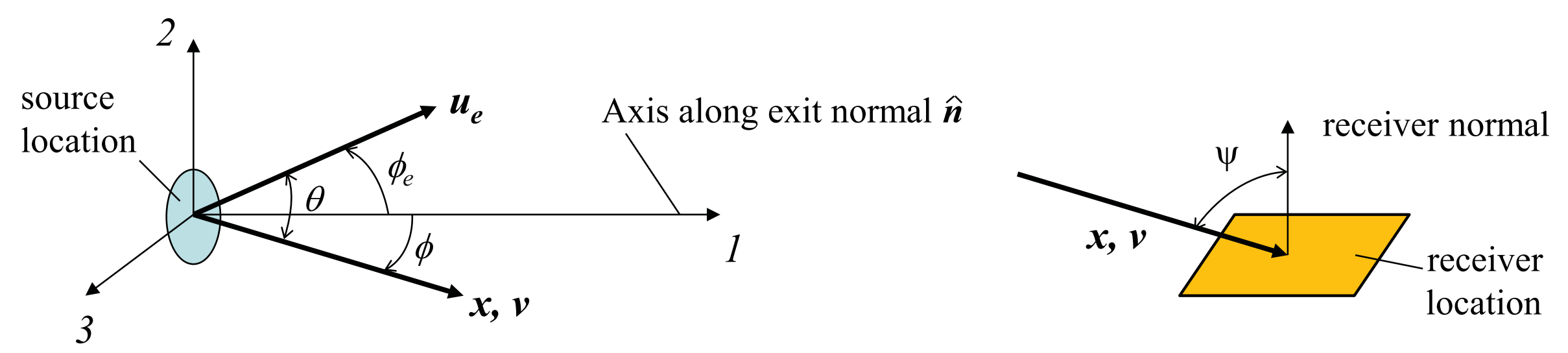

- Subscript $e$ represents exit conditions from source

- Impingement angle with receiver surface given by $\psi$

- Simplifies for axisymmetric conditions

$$
\begin{aligned}
& -\phi_{\mathrm{e}}=0 \\
& -\phi=\theta
\end{aligned}
$$

- other definitions: $s \equiv \beta u_{\mathrm{e}}=\frac{u_{\mathrm{e}}}{\sqrt{2 R T_{\mathrm{e}}}} ; w \equiv s \cos \theta$ 


\section{Plume Model-Steady Mass Flux}

- Can compute many different types of local quantities at receiver position $\boldsymbol{x}$ relative to source

- Steady mass flux given by

$$
\dot{\Phi}(\boldsymbol{x}, t)=\frac{\dot{m} \cos \phi \cos \psi}{A_{1} \pi r^{2}} e^{w^{2}-s^{2}}\left\{\left(w^{2}+1\right) e^{-w^{2}}+\left(\frac{3}{2}+w^{2}\right) \sqrt{\pi} w(1+\operatorname{erf} w)\right\}
$$

- RGA partial pressure measurements typically assume something akin to a vacuum chamber application where gas is at rest

- Actually measures rate at which ions of a given mass/charge ratio successfully navigate quadrupole section after being ionized

- This is a species-selected number flux, so mass flux equation is needed for the ALL device, not plume momentum flux

- Mass flux converted to effective partial pressure using Hertz-Knudsen eqn. 


\section{ALL Device Sensitivity Considerations}

- Expect ALL to distinguish leak constituents against backdrop of ambient environment

- Atmospheric

- Chiefly atomic oxygen $\left(\sim 10^{-8}\right.$ Torr wake-oriented, $>10^{-6}$ Torr opposing ram direction)

- Induced (less affected by ram vs. wake orientation)

- Surface-desorbed, outgassed water vapor (typically $<10^{-8}$ Torr)

- Outgassing of volatile condensable materials (polymerics, organics)

- Discrete events like controlled venting and thruster firings

- Strength depends on surface, source proximity, time on-orbit, etc.

- Review previous space flight mass spectrometer (MS), ion gauge applications

- What issues do/did these instruments contend with? 


\section{Review of Previous Missions (1 of 3)}

- Rosetta ROSINA Instrument Suite

- Rosetta launched 2004, will rendezvous with comet mid-2014

- ROSINA suite features sensitive mass spectrometers capable of high mass/charge ratio resolution

- Capable of detecting species on the $10^{-11}$ mbar level or better

- Suite location, orientation is fixed to the spacecraft

- Attempted to measure interplanetary medium while on-route

- Overwhelmed by spacecraft-induced atmosphere

- Attempts to create direct flux and return flux mass transport models to reproduce measured effects have been unsuccessful 


\section{Review of Previous Missions (2 of 3)}

- Mir Astra-2 Instrument Suite

- Featured an experiment that used an ion gauge to measure the angular distribution of a small Ar cold gas thruster plume

- Ion gauge mounted on arm $(r \sim 1 \mathrm{~m})$ that rotated on a pivot whose axis intersected the nozzle exit center, rotated through $135^{\circ}$ arc

- Always faced nozzle exit

- At highest flow rate setting of about $1 \mathrm{~g} / \mathrm{s}$, could measure angular distribution over four orders of magnitude until surface outgassing effects obscured plume measurements beyond $90^{\circ}$

- Broad plume core region not reproduced by various plume models or DSMC results; thought to have been the result of argon clustering 


\section{Review of Previous Missions (3 of 3)}

- Materials Exposure \& Degradation Experiment (MEDET)

- Undertaken by a group of European research institutions

- Exposed to ambient conditions external to the ISS Columbus module over 19 months

- Ion gauge measured orbit-averaged impingement pressure levels of $10^{-7}$ to $5 \times 10^{-6}$ mbar facing ram direction

- Wake impingement levels were $100-1000 \times$ lower

- Measurements made relative to free stream, not a point source 


\section{Leak Locator Configuration}

- The ALL device operates without physical constraints of these example missions

- Attached to robot arm, positions and orientations not fixed

- Point source location not precisely known

- Raison d'être - reason the device was created!

- Need plume model to understand measurements obtained

- Make use of engineering test setup meant to demonstrate sensitivity of device concept for comparing results with plume model 


\section{Experimental Setup}

- Conducted at NASA Goddard Space Flight Center

- May 2013 and May 2014

- Used a large vertical thermal vacuum chamber featuring shrouds thermally controlled by gaseous or liquid $\mathrm{N}_{2}$

- Capable of reaching $T=100 \mathrm{~K}$

- Special horizontally translating stage built with a platform that could rotate about the vertical axis

- A Stanford Research Systems Model 100 RGA was mounted with the ionizer tip passing through the stage's rotation axis

- Canister enclosed electronics under atmospheric pressure

- To either side were mounted a STABIL-Ion gauge and a Pfeiffer Model PKR 251 full range gauge 


\section{TVAC Configuration}

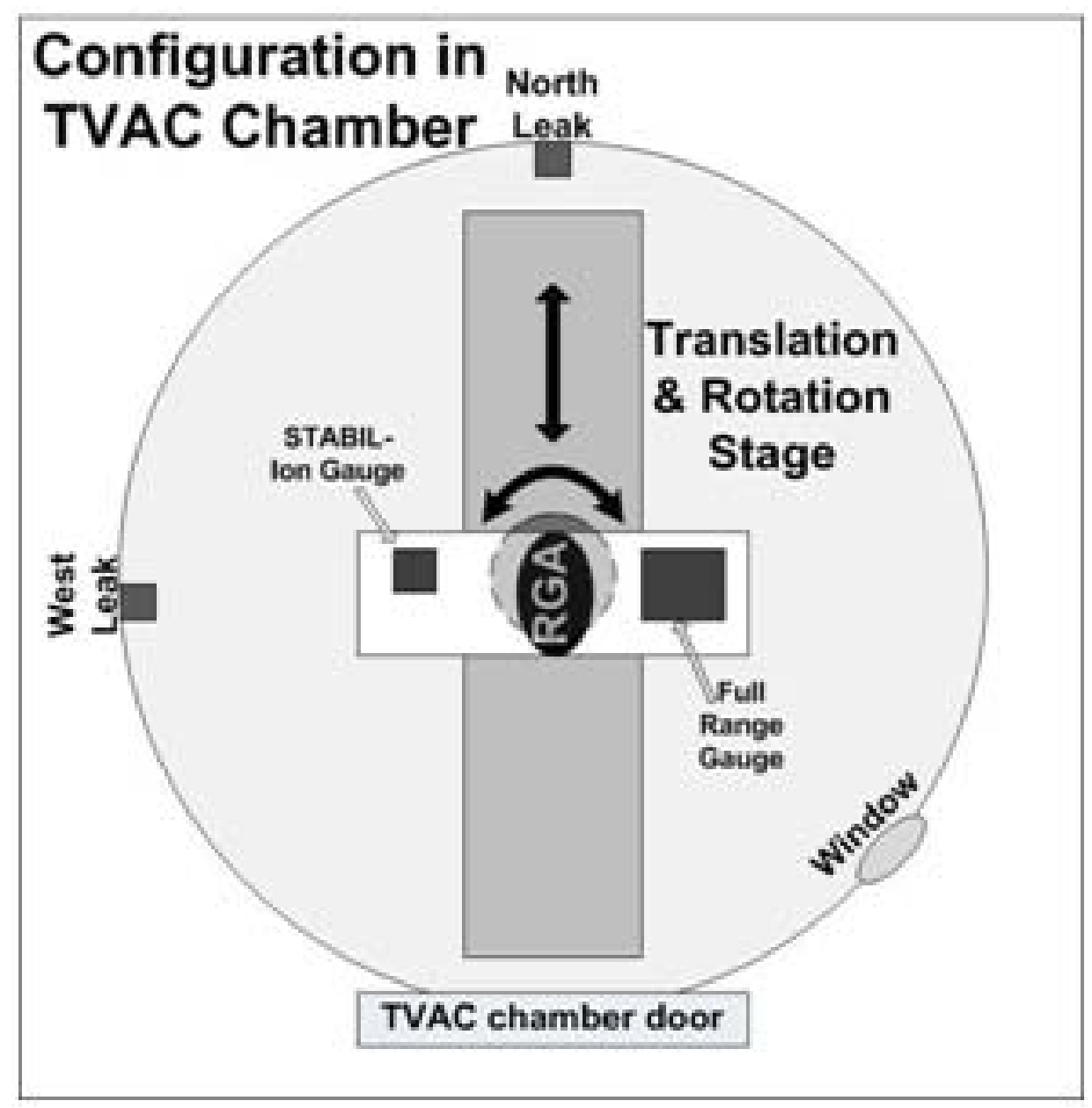




\section{Experimental Setup (3 of 3)}

- A leak source manifold external to the TVAC chamber controlled introduction of gas to either of two orifices

- North Leak (axial relative to translation stage)

- West Leak (oriented transversely)

- Leak tubing inside chamber insulated, thermally controlled with heaters

- Manifold valves were uncalibrated (engineering test consideration)

- High leak rate test cases were measured by weight loss over time from source gas canisters

- Test gases included dry nitrogen, water vapor, and vapor from a strong ammonia solution

- Vapor leaks driven by room temperature equilibrium pressure levels

- $\mathrm{N}_{2}$ used to observe level of agreement between test equipment and TVAC facility pressure measurements 


\section{Engineering Test Plan}

- Leak source types and rates simulated a variety of scenarios

- Ranged from approximately $10 \mathrm{lb}_{\mathrm{m}} / \mathrm{yr}$ to $1 \mathrm{lb}_{\mathrm{m}} /$ day

- Stage translated to a series of set positions along translation axis

- At each position, platform was rotated through a set of angles

- Concentrated on observing RGA response in different modes

- Data reduction effort of most recent runs still underway 


\section{Results-Axial Source $\mathbf{N H}_{3}$}

- Early run contour map shows RGA partial pressure measurements on logarithmic scale based on

- Stage location (vertical axis)

- Rotation angle (horizontal)

- Source located $235 \mathrm{~cm}$ "above" map

- Not quite coincident with translation axis

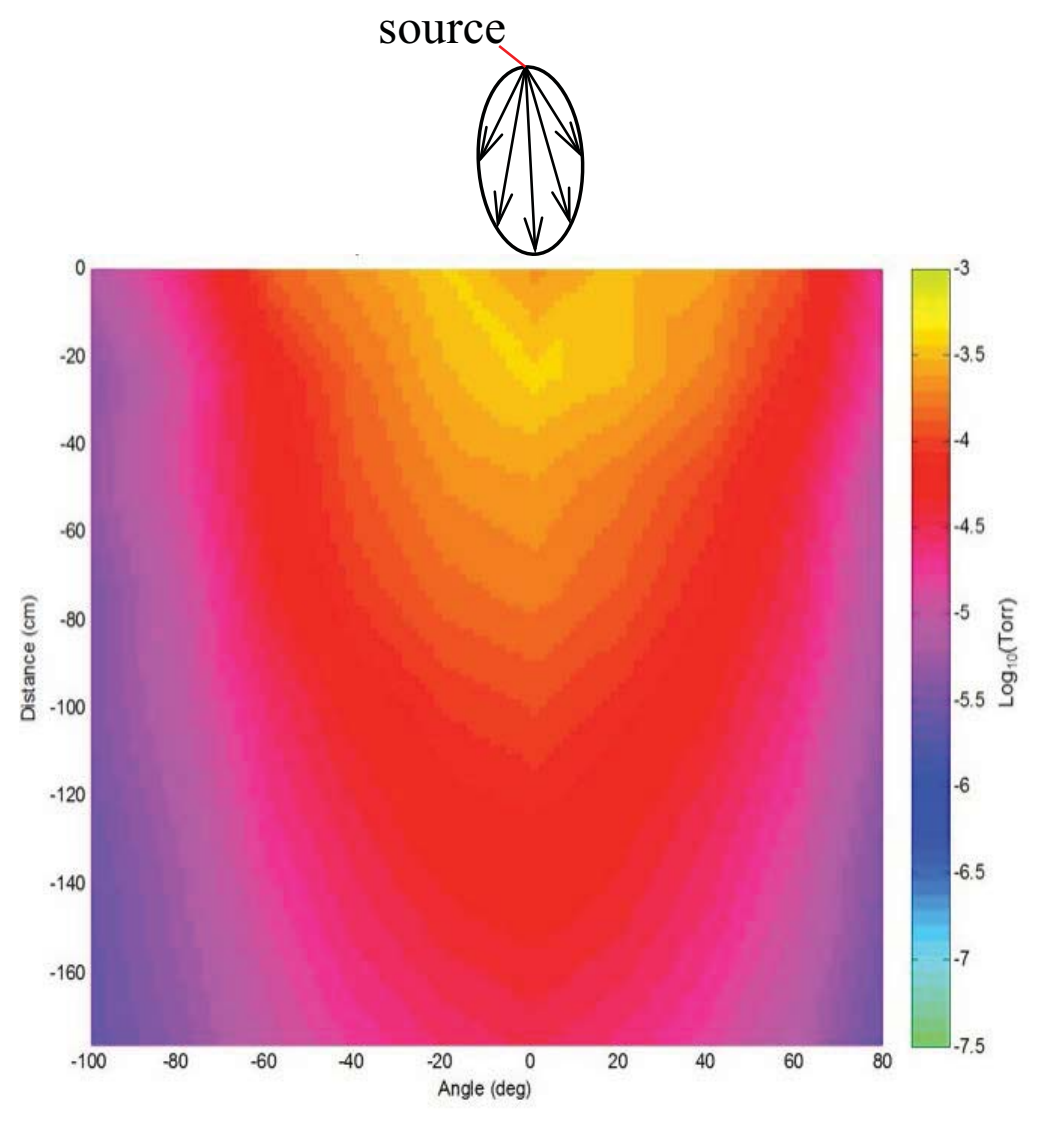

- Mass flow rate $\sim 1 \mathrm{lb}_{\mathrm{m}} /$ day

- Background pressure $\sim 10^{-5}$ Torr 


\section{Results-Axial Source $\mathrm{H}_{2} \mathrm{O}$}

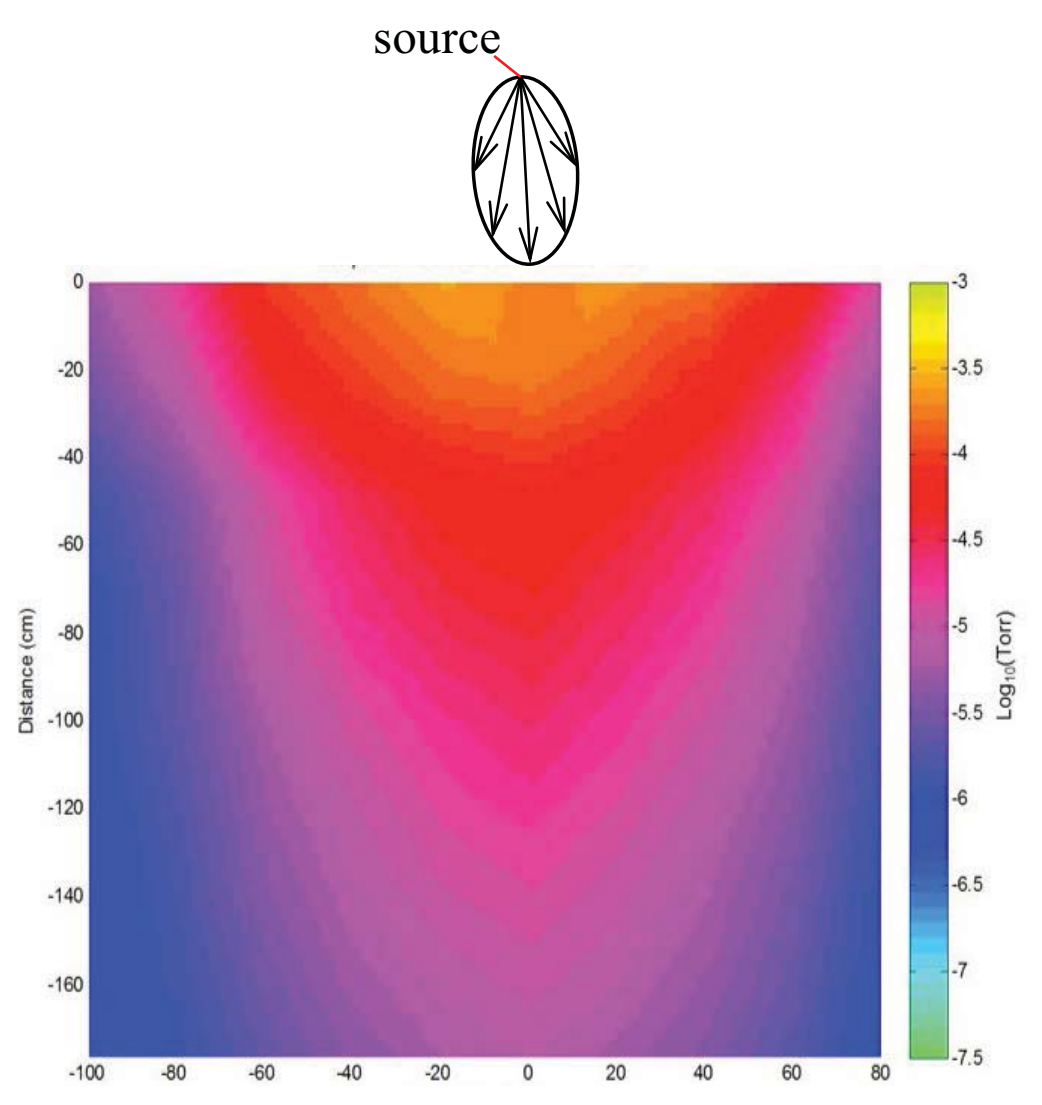




\section{Source Model Comparisons}

- The following runs reflect a subset of data obtained during testing in May 2014

- Better source alignment with translation stage axes than May 2013 testing

- Worked with $\mathrm{H}_{2} \mathrm{O}$ only due to $\mathrm{NH}_{3}$ safety concerns

- Data reduction effort ongoing

- In these runs, facility background pressure measured $\sim 8 \times 10^{-6}$ Torr

- Chiefly air constituents with chamber shrouds operating around $T=100 \mathrm{~K}$

- Detected with facility RGA as well as by test unit

- Model mass flow rate of $\sim 47 \mathrm{lb}_{\mathrm{m}} / \mathrm{yr}, K n=0.02$ based on orifice diameter 


\section{Axial Source Model Comparison}

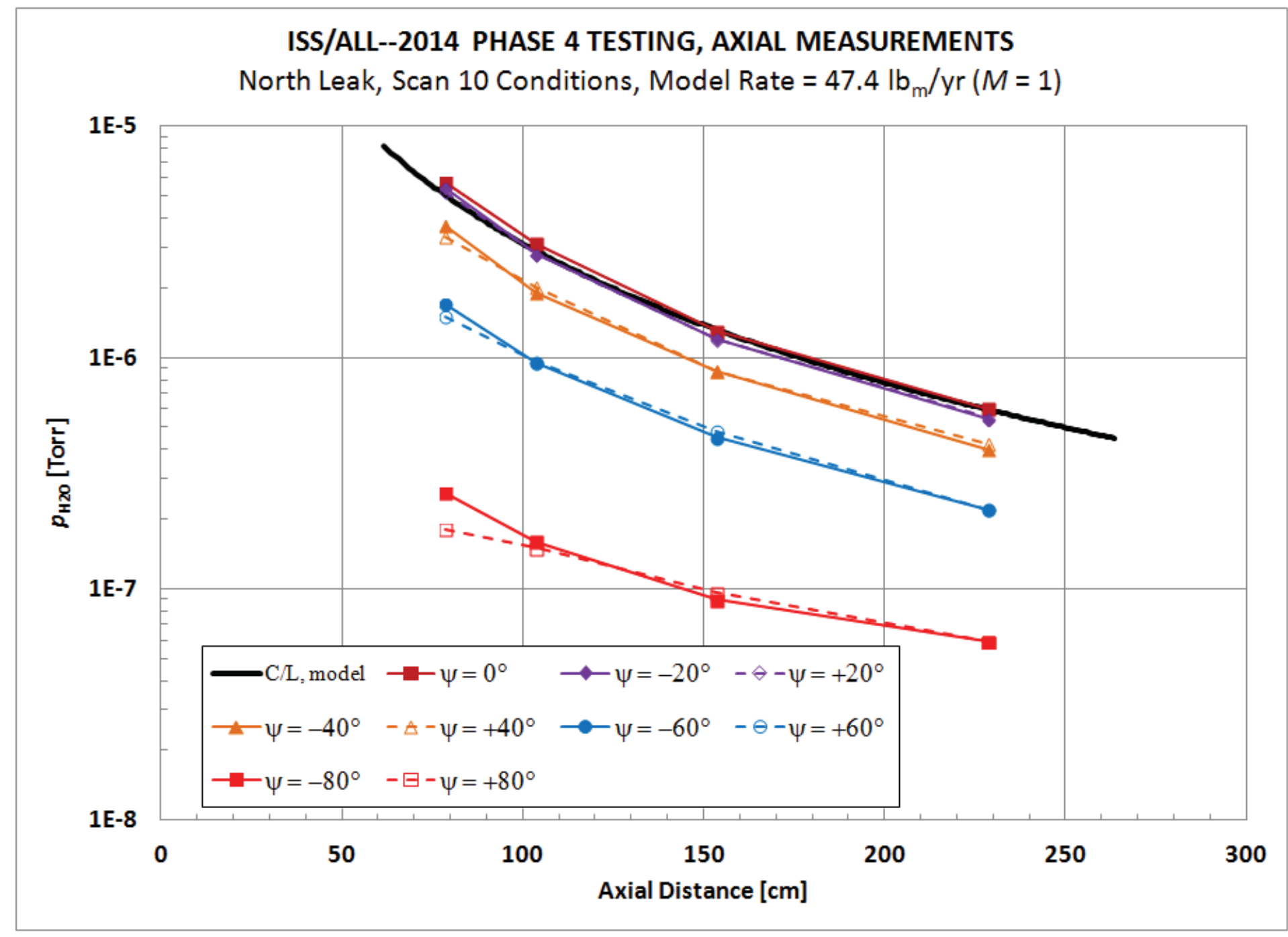




\section{Axial Source Comparison Comments}

- RGA intensity tends to drop off like $1 / r^{2}$ as expected

- Slightly steeper as source is approached

- Could be a scattering effect from portion of platform ahead of instruments

- Dependence on capture angle $\psi$ steeper than cosine

- Assorted safety considerations led to physical features that scatter incident gas at entrance to RGA ionizer section

- Scattering effect dependent on $\psi$ as well as azimuth angle rotating about RGA axis

- Effect needs to be compensated for when devising on-orbit test plan 


\section{Transition Between Sources}

- Leak manifold valves were manipulated to transfer the flow between source locations

- Uncalibrated, so it is uncertain whether flow resistances and resulting rates should have been similar

- Main test emphasis was to demonstrate source detection

- RGA then translated in $25 \mathrm{~cm}$ increments across range of motion

- At each stop, measurements were obtained at various platform rotation angles over a range of $100^{\circ}$

- Transverse source located $\sim 1.5 \mathrm{~m}$ off of translation stage axis

- This setup emulates an operation where ALL is translated across a suspect surface in a plane containing a leak 


\section{Transverse Source Measurements}

- Used axial run at same main valve setting to obtain $\psi$ dependence

- Measurements exhibit a fair amount of uncertainty not seen in axial run

- Divide measurements to obtain estimates for free expansion of transverse plume

- Peak values apparently offset about $10^{\circ}$ despite pre-test alignment

- Thermally-induced mechanical stresses during cooldown?

- Ice formed on orifice tip?

- Less likely 


\section{Transverse Source Comparison}

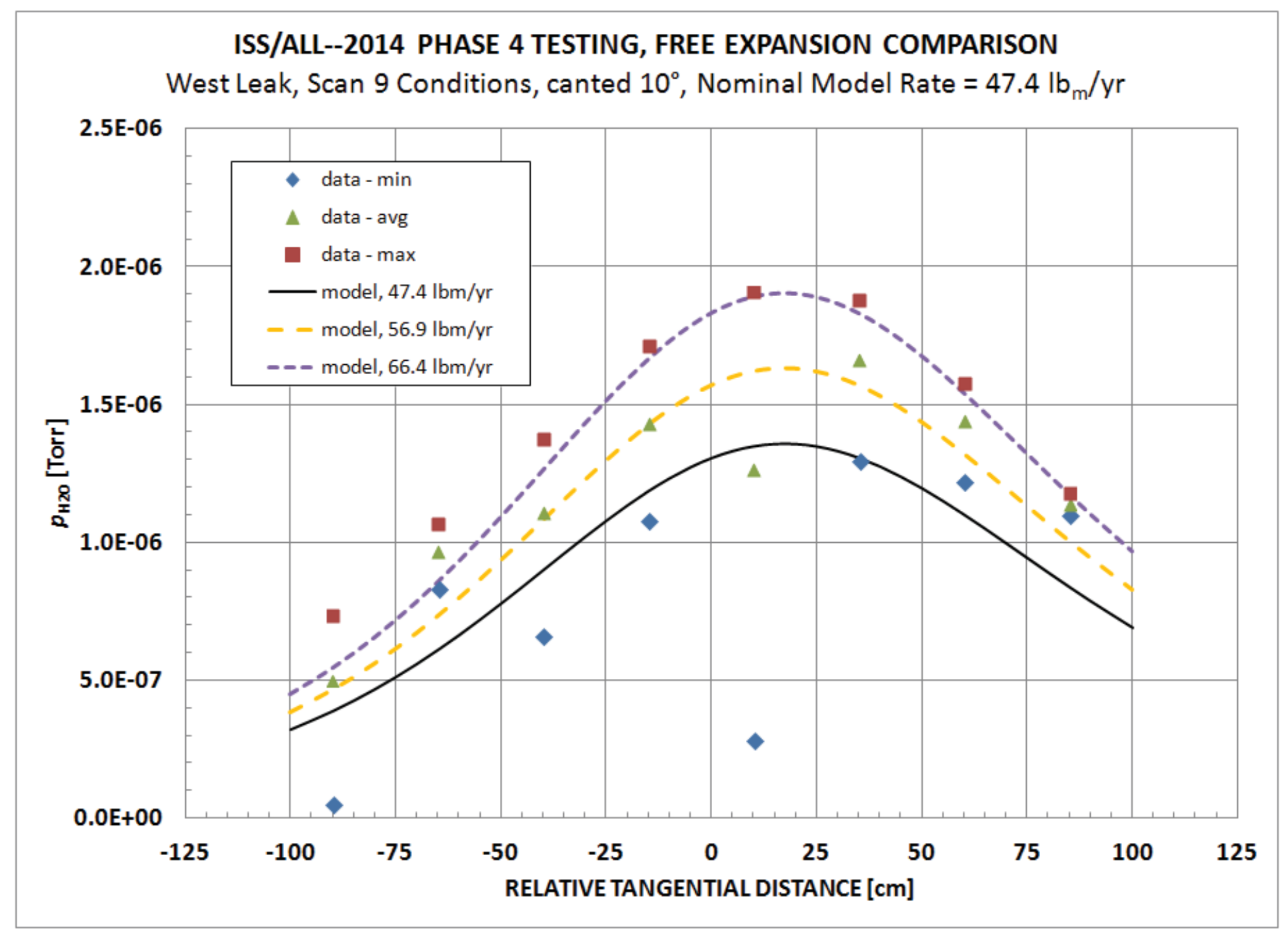




\section{Transverse Source Comparison Cmts.}

- Model results calculated for same $47 \mathrm{lb}_{\mathrm{m}} / \mathrm{yr}$ sonic orifice appear to fit lower range of measurements

- Black solid line in both result figures

- Same conditions but with $20 \%$ and $40 \%$ higher mass flow rates fit middle and upper ranges of RGA measurements

- Model appears to capture curvature of source angle dependence also

- Can create model of approximate intersection of RGA path with transverse source plume 


\section{RGA Path, Plume Intersection Estimate}

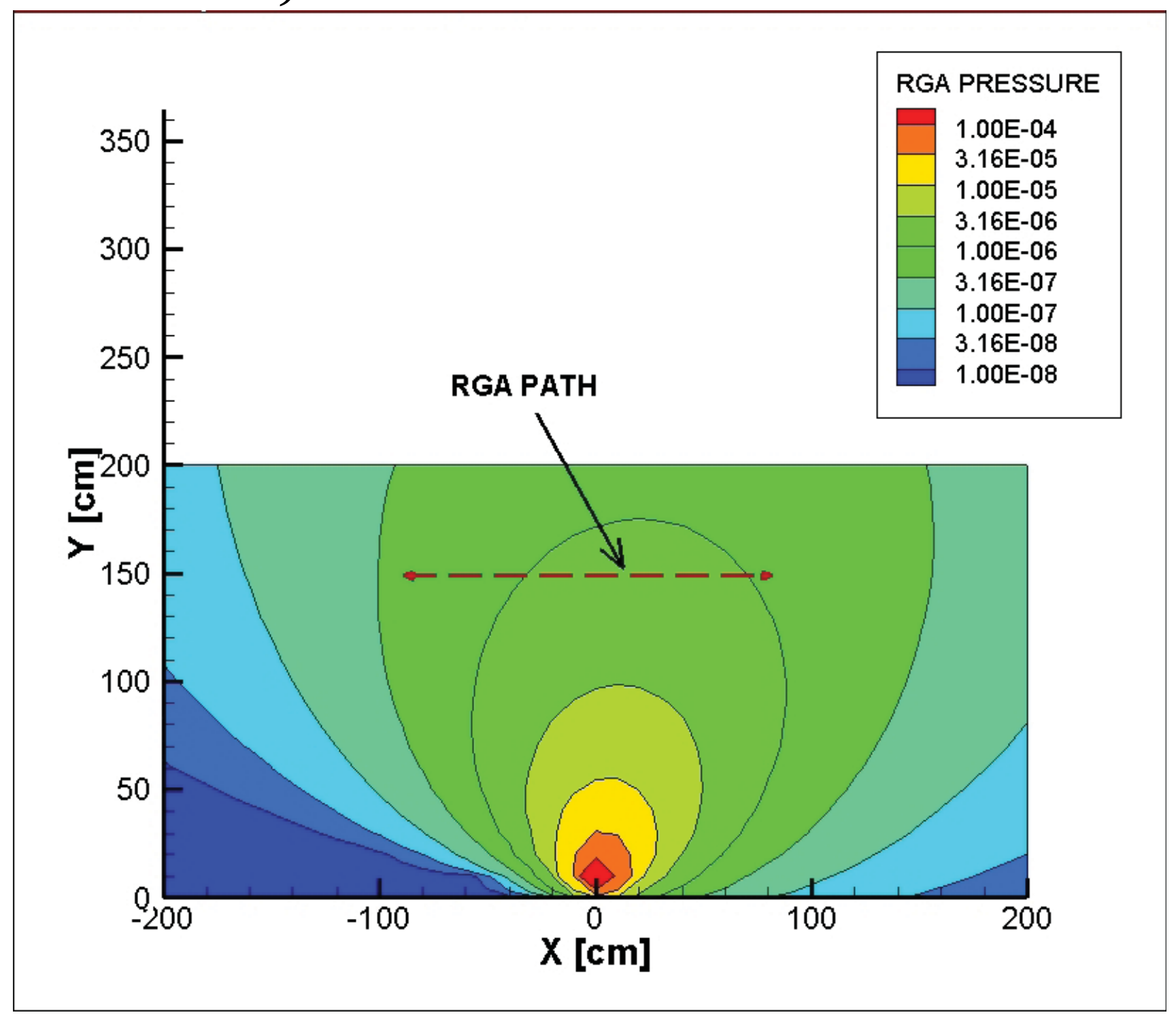




\section{Concluding Remarks}

- The COTS RGA unit performed well

- Displayed desired levels of sensitivity to the intensity of faint leak sources

- Demonstrated "directionality," or sensitivity to pointing angle

- Non-cosine dependence will need to be accounted for when developing testing and operational plans at ISS

- Sufficiently robust when adapted to high vacuum and extreme thermal environments

- Plume model did a reasonable job of reproducing test conditions for axially and transversely located sources

- The ALL device has been selected to demonstrate its capabilities at the ISS 


\section{Acknowledgments}

- The authors wish to thank all of the team members on the Ammonia Leak Locator project for their contributions throughout the project, the NASA GSFC Satellite Servicing Capabilities Office, NASA GSFC thermal vacuum facility employees, and the JSC Engineering Directorate. Additional thanks to Universal Cryogenics, who designed and built the Translation \& Rotation Stage for use in this cryo-vacuum test.

- The authors also wish to thank our presenter for his efforts and graciousness. 